\title{
Performance Enhancement of Cognitive Radio Networks Using SINR-Based Cooperative Beamforming
}

\author{
P. Suresh Kumar, G. Nirmala, S. Manimegalai, H. Arulvedi \\ ${ }^{1}$ Department of EEE, Mahendra Engineering College (Autonomous), Mahendhirapuri, \\ Mallasamudram - 637 503, Tamil Nadu, India. \\ 2Department of ECE, Mahendra Institute of Technology, Mahendhirapuri, \\ Mallasamudram - 637 503,Tamil Nadu, India. \\ 3 Department of ECE, Mahendra Engineering College for Women, \\ Tiruchengode, Namakkal 637205, India \\ ${ }^{4}$ Department of EEE, Mahendra Engineering College for Women, \\ Tiruchengode, Namakkal 637205, India
}

\begin{abstract}
The Model Cognitive Radio Network (MCRN) is developed as a cooperative beam forming method specially designed to provide dynamic spectrum allocation for Primary and Secondary Users. The purpose of the design is to minimize the transmit power of a transmitter area, an eavesdropper, a primary receiver, and multiple secondary receivers were given with distinct Signal to Interference and Noise Ratio (SINR). This method easily detects noises without any prior knowledge about the signal. The signal fading is avoided using the technique was relay between the users, this leads to result in the reduction of outage probability. The performance estimation of the proposed optimal approach is validated and compared using the computer simulations software with an accuracy of $99.8 \%$ of the existing methods.
\end{abstract}

KEYWORDS: Cognitive Radio Network (CRN), cooperative beamforming, physical layer security, transmit power, Signal to interference and noise ratio (SINR), signal fading

\section{INTRODUCTION}

The Cognitive Radio (CR) is an impressive modern technology that has the makings to deal with the forceful requirements and advantages of the radio spectrum in the next generation of wireless communications [1-4]. In this classical approach, the primary users allow secondary users to access the spectrum were licensed to primary users (PUs) only when the spectrum is not used in current scenario[5-9].This method leads to cooperative communication for Cognitive Radio Networks (CRN) has been considered as a new CR standard of method. According to cooperative communications standard methodology, primary network leases the part of the spectral resources to SUs in exchange for cooperation techniques [10-14]. In this method of information with beam-form designing, the nodes with Multi Input Single Output (MISO) are considered as a secondary network whose target is to improve the secret rate of single input single output (SISO) primary network sources. It also investigates the cooperative spectrum access for SISO CRNs, which indicates to improve the secure transmission of PUs via cooperating SUs. However, both of this [15] do not consider the physical layer security of SUs.
On the other hand, improving physical layer security based on Artificial Noise (AN) was proposed [16] in this way [10], where a non-zero secret rate of output could be achieved, even when the channel condition of legitimate user was worse than that of eavesdropper (Eve) secrecy capacity improvement techniques. In this method, several works have been proposed to study the physical layer security based on AN. But signal to interference and noise ratio (SINR) based beam forming was proposed to provide a legitimate user and eavesdropper with different requirements of SINR. The new method has been depicted based on joint optimization of both information covariance [17] and covariance for SU in CRNs. However, this joint information beam forming and Beam forming techniques are to improve the physical layer security in CRNs for both SUs and PUs has not been studied. In this proposed work, a new CRN technique has been discussed and where the both PU and SU transmit confidential information to intended receivers. The main objective of the present study is to provide intended users with different SINR, and to design joint information beam forming and $\mathrm{AN}$ beam forming at the secondary transmitter. It has been 
proved that using Semi Definite Relaxation (SDR), the initial non convex optimization can be converted into a convex semi definite programming (SDP) method, which can be efficiently solved with the existing techniques and validated with earlier methods [18].

\section{WIRELESS COMMUNICATION}

The new era of Wireless technology is spreading on the double, and the understanding of universal wireless computing and communications offer the commitment of many societal and individual benefits. The Consumer devices such that PDAs, mobile phones, and portable computers pocket intense were attentiveness. This impact of wireless technology is widespread over the entire world with cutting edge techniques. This ignition method creates an escalating demand for more radio spectrum waves. However, most easily usable spectrum bands have allocated, even though many studies have shown that these bands are considerably wasted in this methodology [19]. These evidences have drawn the search of new method for notable progress in radio technologies. This can be fit for future demands and in terms of efficiency with spectrum analysis and performance of the PDA applications. The cognitive radio system was given the assurance of a disruptive technology with innovations in future wireless world [20].

\section{A. Cognitive Radio Network}

A cognitive radio is a knowledgeable radio that can be programmed and configured dynamically. It is designed to produce the best wireless channel. Such that a radio wave detects the available channels automatically and changes its transmission or reception parameters to permit more wireless communications simultaneously [21].

Cognitive Radios are classified into two main types based on its transmission and reception parameters. They are classified as: Full cognitive radio (Mitola Radio) and Spectrum-sensing cognitive radio. Dependent of the spectrum available for cognitive radio are classified as Licensed-Band Cognitive Radio, Unlicensed-Band Cognitive Radio, Spectrum mobility, Cognitive radio networks, Spectrums sharing cognitive radio networks and Sensing-based Spectrum sharing cognitive radio networks

DSA (Dynamic Spectrum Access) networks were caused numerous challenges in wireless communications and networking. The first task of radio waves could be operated in multiple frequency bands of medium. But these radios were designed for a particular band of frequency was not optimum. The radio waves can choose one of the frequency available bands; the users had to pick the right band for those particular applications. The band of frequency selection was typically by a two-step process called radio waves that to determine the appropriate FCC rules, and then radios need to decide which band is the most suitable for usage. The wireless devices had to synchronize on the realization of various physical links and network layer functions that makes the use of the best available spectrum. This cognitive Radio Networks were to be a perfect fit to realize the above functionality [22].

The CRNs should sense their operating environments and also modify with their implementations. It included the signal propagation environment, node density, traffic load, mobility, and DSA networks available with spectrum. This generation wireless networks (e.g., Wi-Fi) use very restricted forms of cognitive optimization with rate adaptation and spectrum agility with channel selection. But this needs much more aggressive adaptation, called a wider spectrum bands and more radical runtime protocol optimizations to improve the spectrum efficiency and wireless network capacity.

There has been a significant amount of research in CRNs, looking at adaptation of physical mode of modulation and coding, link of adaptive MAC protocols and network collaborative network formation layer. CRNs desired a radio device that has very malleable with various protocol functions that changed completely at runtime. A softwaredefined radio was flawless platform for CRNs and radios some MAC protocol functions into action using hardware. Which limits the degree of runtime flexibility to a small predefined set of changes with chosen between a handful of transmission rates. On the other hand, SDRs attempt to do much processing in the digital domain with limitations of analog/digital converters. Digital processing capacity and power constraints were fusion of analog and digital processing is still used. An analog circuit converts the signal between the radio carrier frequencies and an intermediate frequency.

Intermediate frequency of signal is digitized using software were changed at runtime. Even the analog circuits are designed to be flexible. SDR software controls the oscillator frequencies, receiver attenuation, transmit power, filter Centre frequencies, and receiver gain. Cognitive networks do not strictly need SDRs, the flexibility offered by SDRs is very attractive, prototyping and evaluating cognitive networking technology. A CRN combined with multiple information sources to accommodate their transmission waveforms, channel access method, and networking protocols [23]. 


\section{B. Technology Used in Cognitive Radio Network}

Cognitive radio was a software-defined radio extension; center of attraction is spectrumsensing cognitive radio. The problem in spectrumsensing cognitive radio is designed with high quality spectrum sensing devices and algorithm nodes. The information has to be exchanged between nodes for sophisticated spectrum sensing techniques. The probability of false detection had decreased by increasing the number of cooperating sensing nodes. Orthogonal Frequency-Division Multiple Access (OFDMA) has used as a multi-user version of the orthogonal frequency-division multiplexing (OFDM) digital modulation scheme. This Multiple access is achieved in OFDMA by assigning with subsets. The individual user's node is shown in figure 1.

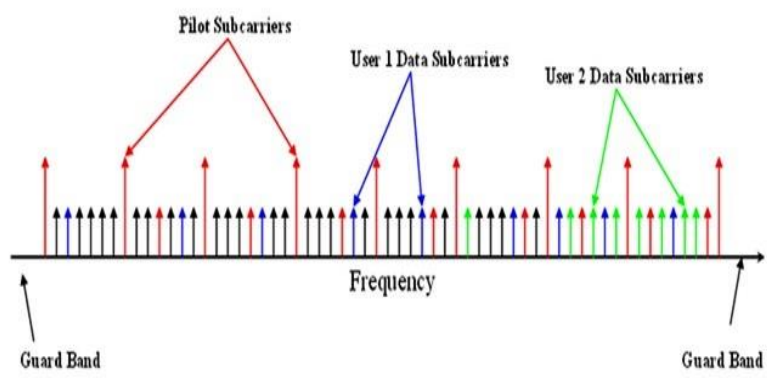

\section{Fig.1 OFDMA Subcarrier of Cognitive Radio} Network

\section{Secrecy Problem in Wireless Environment}

The progress in wireless communication technology connected to the network anywhere, anytime. However, this ease of accessibility makes it easy to communication over the medium, thus promoting privacy concerns. Secrecy problems involve three nodes: transmitter, receiver and an eavesdropper. Secrecy problem due to an eavesdropper was referred as passive attack. The emergence of large-scale, dynamic and decentralized wireless network imposed with new challenges on classical security measures such as cryptography. Security technologies were required to complement the cryptography and significantly improve the overall security issues in wireless communication network.

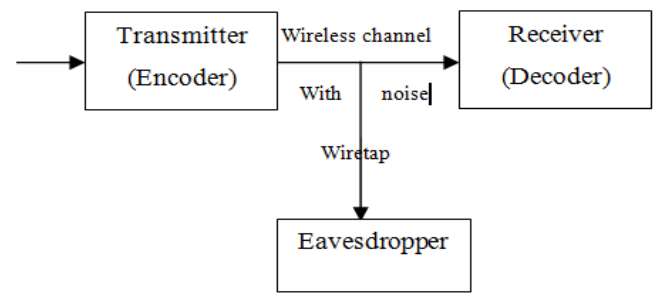

Figure.2 Wiretap Channel Model in Wireless Environment
The physical layer security of wiretap channel model is illustrated by the above figure.2. The secure communication framework does not account for the physical reality of communication channels. This shown of a more realistic communication model, now known as the wiretap channel, where noise in the main channel and eavesdropper's channel is introduced obviously. The eavesdropper is assumed to be passive and uncertain to the transmitter.

\section{Physical layer Secrecy}

The physical layer or layer 1 is the first (lowest) layer in the 7-layer open system interconnect (OSI) model of the computer networking. It is commonly abbreviated as PHY. PHY have capacity for

1. Definition of hardware specifications

2. Encoding and scaling

3. Data transmission and reception

4. Topology and Physical network design

In all communication systems, the upper layers of the protocol stack handle the authentication concern, confidentiality and privacy using variations of private-key and publickey cryptosystems [24]. Nowadays many results from information theory, signal processing, and cryptography recommend that there is much security to be gained by accounting for the imperfections of the physical layer while designing secure systems. To achieve security in PHY, there are multiple approaches such as

1. Preprocessing scheme

- Coding

- Key generation

- Artificial noise scheme

2. Game theoretic scheme

3. Signal processing

4. Cooperation communications

5. Many others

In the last decade, multi-antenna techniques have been extensively investigated from the perspective of providing high throughput and reliable communications. Recently, there has been growing interest in using multiple antennas to achieve secure communication, which is known as Physical Layer Secrecy. The idea of physical-layer secrecy is to add structured redundancy in the transmit signal such that the legitimate user can correctly decode the confidential information, but the eavesdroppers can retrieve almost nothing from their observations [25].

To make physical-layer secrecy, a practical approach, the channel condition of the legitimate 
user should be better than the eavesdropper's channel condition. However, in practice, this may not be possible always. To ease the dependence on the channel's conditions, main focus of the recent studies is mainly on multi-antenna transmission, since multiple transmit antennas provide spatial degrees of freedom to degrade the reception of the eavesdroppers. A possible way to do this is transmit beam forming technique, where the transmit signal concentrates on the legitimate user direction while at the same time reduces power leakage to the eavesdropper. Apart from this, a more active approach is to send artificially generated noise to interfere the eavesdroppers purposely. The way of generating AN depends on with the transmitter knows the eavesdroppers channel state information.

\section{E. Beam forming}

Beam forming is a general signal processing technique used to control the directionality of the reception or signal transmission on a transducer array. Using beam forming we can direct the majority of signal energy we transmit from a group of transducers in a chosen angular direction (or) we can calibrate the group of transducers while receiving signals such that we primarily receive from a chosen angular direction.

Beam forming techniques can be broadly classified into two categories.

1. Conventional (fixed (or) switched beam) beam formers

2. Adaptive beam formers (or) phased array

- Desired signal maximization mode

- Interference signal minimization or cancellation mode

Here the cooperative beam forming technique to reduce energy consumption as well as interference.

The basic point in beam forming is, when we set multiple transducers next to each other sending out signals, we will get some kind of interference pattern, just like we see in a pond when we throw several stones in at once and create interfering ripples. If the selected the spacing between the transducers and the signal delay of the transducer, This can create an interference pattern, in particular, in which the majority of the signal energy goes out in one angular direction. While transmitting, to change the directionality of the array at each transmitter, a beam former controls the phase and relative amplitude of the signal, in order to create a pattern of constructive and destructive interference in the wave front. While receiving, the gained information of different sensors is combined in such a way that the expected radiation pattern is observed preferentially.

\section{F. Cooperative Beamforming}

The beamforming is used as a cooperation technique on both transmitter and receiver side. Cooperative beam forming is a simple technique consisting of decimating the number of cooperating nodes in virtual antenna array (VAA) with the aim of reducing energy consumption as well as interference. In this technique randomly distributed nodes in a network cluster form an antenna array and beam form data to a faraway destination without each node exceeding its power constraint. The proposed scheme is based on the idea of cooperative beam forming which reduces the time required for information sharing. The main idea used here is different source nodes in the network are allowed to transmit simultaneously. The collaborating nodes will receive linear mixture of the transmitted packets. Subsequently, each collaborating node transmits a weighted version of its received signal. The weights are such that one or multiple beams are formed, each focusing on one destination node, reinforces the signal intended for a particular destination, as compared to other signals. Each collaborating node computes its weight based on the estimated channel coefficients between sources and itself. This scheme achieves higher throughput, lower delay, lower SINR. Hence cooperative beam forming is used to improve the quality of communications.

\section{III.SYSTEM MODEL AND PROBLEM FORMULATION}

The system model and formulate the proposed secret transmission beam forming designed with following methods. The weights are such that one or multiple beams are formed, each focusing on one destination node, reinforces the signal intended for a particular destination, as compared to other signals. Each collaborating node computes its weight based on the estimated channel coefficients between sources and itself.

\section{A. System Model}

Consider a CR system having a primary transmitter (PT), a primary receiver (PR), an eavesdropper (Eve), a secondary transmitter (ST), and multiple secondary receivers (SRs). It is implemented that there are many $(M>1)$ SRs, denoted by the set $K_{M}=\{1 \ldots . ., M\}$. ST is armed with many $(N>1)$ antennas and all other nodes are equipped with single antenna.

The baseband equivalent channels from PT to PR, Eve and SRs are symbolized as $g_{p} \in \mathrm{C}^{1 \times 1}, g_{e} \in$ 
$\mathrm{C}^{1 \times 1}$ and $g_{s, m} \in \mathrm{C}^{1 \times 1}$ where $\quad m \in K_{M}$, respectively. The channels from ST to PR, Eve and SRs are denoted as $h_{p} \in \mathrm{C}^{N \times 1}, h_{e} \in \mathrm{C}^{N \times 1}$ and $h_{s, m} \in \mathrm{C}^{N \times 1}$ where $m$ $\in K_{M}$, respectively. It is assumed that the channels are circularly symmetric complex Gaussian (CSCG) random variables, which are perfectly known. The secondary network can access the spectrum licensed to PUs when the physical layer security of primary network is guaranteed. To provide the strongest distortions to Eve, AN is used by ST. A possible way to do this is transmit beam forming technique, where the transmit signal concentrates on the legitimate user direction while at the same time reduces power leakage to the eavesdropper.

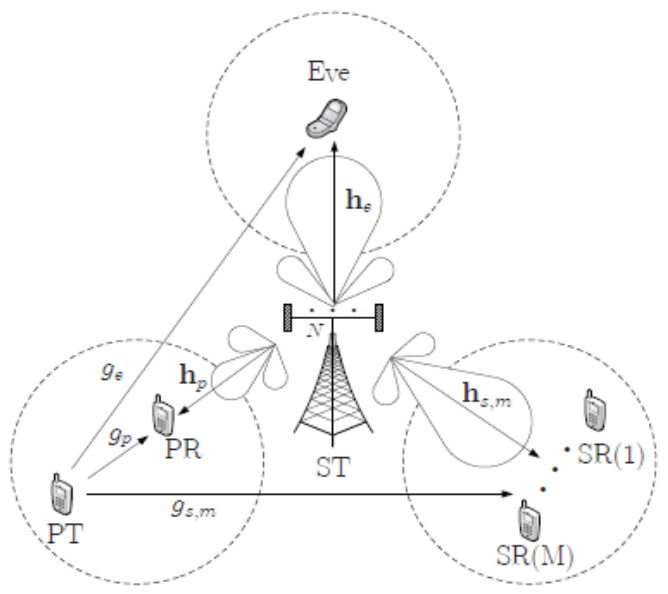

Fig.3 Spectrum leasing for physical layer secrecy via SINR-based cooperative beam forming

The baseband transmitted signal at ST can be expressed as

$x_{s}=\sum_{m \in K M} s m v m+w v e$,

where $v_{m} \approx C N(0,1)$ is the signal transmitted to the $m$ th $\mathrm{SR} ; v_{e} \approx C N(0 ; 1)$ is the AN signal transmitted to Eve; $s m$ and $w$ are information beam forming vectors and AN beam forming vector respectively. Assuming that the transmit power of PT is $P p>0$ and the baseband transmitted signal at PT is $v_{p} \approx$ $C N(0 ; 1)$, the received signal at PR, Eve and SRs can be expressed respectively as,

$$
\begin{aligned}
& y_{p}=\sqrt{P_{p}} g_{p} v_{p}+h_{p}^{H} x_{s}+z_{p} \\
& y_{e}=\sqrt{P_{p}} g_{e} v_{p}+h_{e}^{H} x_{s}+z_{e} \\
& y_{s, m}=\sqrt{P_{p}} g_{s, m} v_{p}+h_{s, m}^{H} x_{s}+z_{s, m}
\end{aligned}
$$

This explained the appropriate $p$ to eliminate such unfavorable case. This was assumed that P1-SDR is always feasible.

\section{B. The Proposed Design Formulation}

To minimize the transmit power of ST, while providing Eve, PR and SRs with different SINR, the power minimization problem can be formulated as

$$
\begin{aligned}
& P 1: \min _{\{s m, V m \in \mathrm{K} m\}, w} \sum_{m \epsilon K m}\left\|s_{m}\right\|^{2}+ \\
& \|w\|^{2} \text { s.t } \frac{P_{p}\left\|g_{p}\right\|^{2}}{\sum_{m \in K m} \| h_{p}^{H} s_{m}||^{2}+|| h_{p}^{H} w||^{2}+\sigma_{p}^{2}} \geq \gamma_{p} \\
& \frac{P_{p}\left\|g_{e}\right\|^{2}}{\sum_{m \in K m} \| h_{e}^{H} s_{m}||^{2}+\left.|| h_{e}^{H} w\right|^{2}+\sigma_{p}^{2}} \leq \gamma_{e, p} \\
& \frac{\| h_{s, m}^{H} s_{m}||^{2}}{\left.\sum_{m \in K m}|| h_{s, m}^{H} s_{i}\right|^{2}+|| h_{s, m}^{H} w||^{2}+\left.P_{p}|| g_{s, m}\right|^{2}+\sigma_{s, m}^{2}} \geq \gamma_{s} \\
& \frac{\| h_{e}^{H} s_{m}||^{2}}{\sum_{i \neq m, i \in K m}|| h_{s, e}^{H} s_{i}||^{2}+|| h_{e}^{H} w||^{2}+P_{p}|| g_{e} \|^{2}+\sigma_{e}^{2}} \leq \gamma_{e, s}
\end{aligned}
$$

where $\gamma_{p}>0$ is the minimum SINR requirement for PR; $\gamma_{e, p}>0$ is the maximum allowable SINR threshold for Eve to eavesdrop the information between PT and PR; $s>0$ is the minimum SINR requirement for all SRs; $\gamma_{e, s}>0$ is the maximum allowable SINR threshold for Eve to eavesdrop the information between ST and SRs.

\section{OPTIMAL SOLUTIONS}

Using SDR, P1 can be relaxed as the following SDP.

$$
\begin{aligned}
& \frac{1}{\gamma s} \operatorname{Tr}\left(H_{s, m} S_{m}\right)-\sum_{i \neq m, i \in k M} \operatorname{Tr}\left(H(s, m) S_{i}\right)- \\
& \operatorname{Tr}(H s ; m W m) \geq P p\|g s ; m\|^{2}+\sigma_{s, m}^{2} \\
& \frac{1}{\gamma e s} \operatorname{Tr}\left(H_{e} S_{m}\right)-\sum_{i \neq m, i \in k M} \operatorname{Tr}\left(H(s, m) S_{i}\right)- \\
& \operatorname{Tr}(H e W) \leq P p\|g e\|^{2}+\sigma_{e}^{2}
\end{aligned}
$$

where $\quad S_{m} \triangleq S_{m} S_{m}^{H}, \quad \mathrm{~W} \triangleq w w^{H}, H_{p} \triangleq h_{p} h_{p}^{H}, H_{e} \triangleq$ $h_{e} h_{e}^{H}$ and $H_{9, m} \triangleq h_{s, m} h_{s, m}^{H}$.

Thus, P1-SDR generally provides suboptimal solutions for P1. Nevertheless, can be proved the optimality of SDR by showing the optimal solutions for P1-SDR must be rank-1.

This explained the appropriate $p$ to eliminate such unfavorable case. This was assumed that P1-SDR is always feasible. Since P1-SDR is convex, the Lagrange of P1-SDR can be defined as the optimal jamming and the optimal information co variances $\forall m \in K_{M}$ satisfy $\operatorname{Rank}\left(S^{*} m\right)=1$. The SDR is optimal for P1. Thus, one can obtain the optimal solutions by solving P1-SDR. Remarkably, P1- SDR is a standard SDP problem and can be efficiently solved using CVX tools. This cooperative 
beam forming is used to improve the quality of

\section{communications.}

\section{SIMULATION RESULTS}

The performance of the proposed optimal approach is evaluated by using computer simulations. We set $\mathrm{P}_{\mathrm{p}}=30 \mathrm{dBm}, \gamma_{p}=10 \mathrm{~dB}, \gamma_{e, p}=0$ $\mathrm{dB}$ for primary users. The channel vectors $g_{p}, g_{e}$, $\mathrm{g}_{\mathrm{s}, \mathrm{m}}, \mathrm{h}_{\mathrm{p}}, \mathrm{h}_{\mathrm{e}}$, and $\mathrm{h}_{\mathrm{s}, \mathrm{m}}$ and the receive noises $\mathrm{z}_{\mathrm{p}}, \mathrm{z}_{\mathrm{e}}, \mathrm{Z}_{\mathrm{s}, \mathrm{m}}$ are generated as independent CSCG random variables. Zero forcing beam forming based algorithms were also provided for comparison and validation of results.

\section{A. For ST power consumption $\left(P_{s}\right)$ Vs SINR requirement $\left(\gamma_{s}\right)$}

Figure 4 illustrating the Secondary user (SU) have saving more power. The ST power consumption increases with increasing SINR requirement and increasing SRs. This is because more power will be used to satisfy minimum SINR requirement for SRs.

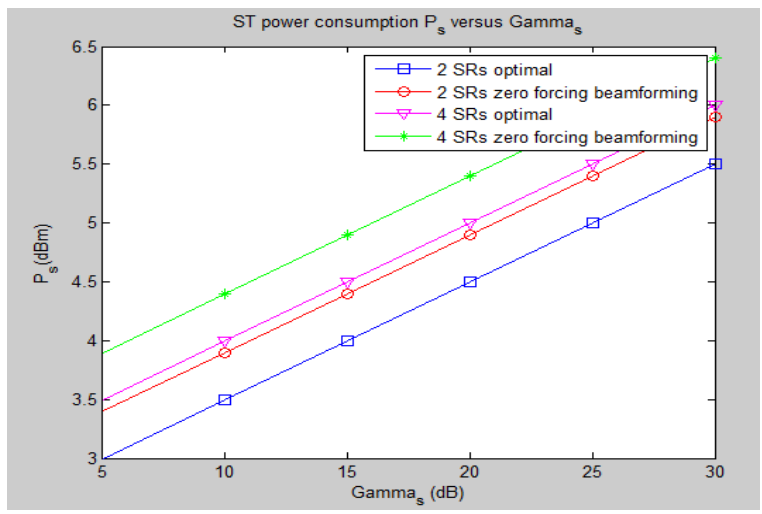

Figure.4 ST Power consumption $P_{s}$ versus $\gamma_{s}$ with $P_{\mathrm{p}}=30 \mathrm{dBm}, \gamma_{p}=10 \mathrm{~dB}, \gamma_{e, p}=0 \mathrm{~dB}, \gamma_{e, s}=0 \mathrm{~dB}$.

\section{B. For $S T$ power consumption $\left(P_{s}\right)$ Vs SINR requirement $\left(\gamma_{e, s}\right)$}

Since Ps-SDR is convex, the Lagrange of P1-SDR can be defined as the optimal jamming and the optimal information co variances $\forall m \in K_{M}$ satisfy $\operatorname{Rank}\left(S^{*} m\right)=1$. The SDR is optimal for P1. Thus, one can obtain the optimal solutions by solving P1-SDR. Remarkably, P1- SDR is a standard SDP problem and can be efficiently solved using CVX tools.

Figure.5 illustrated the ST power consumption decreases with $\gamma_{e, s}$. This is because SINR constraints can be satisfied with smaller ST power consumption with increasing of $\gamma_{e, s}$.

Hence, the value of $\gamma_{s}$ decreases, there is a considerable power reduction. The ST power consumption decreases with $\gamma_{e, s}$. This is because
SINR constraints can be satisfied with smaller ST power consumption with increasing of $\gamma_{e, s}$ C. Improving security in Cognitive Networks using relay scheme

The outage probability is reduced between secondary transmitter and secondary receiver using relay scheme. Since P1-SDR is convex, the Lagrange of P1-SDR can be defined as the optimal jamming and the optimal information co variances of $\operatorname{Rank}\left(S^{*} m\right)=1$. The SDR is optimal for P1. Thus we can obtain the optimal solutions by solving P1-SDR. Remarkably, P1- SDR is a standard SDP problem and can be efficiently solved using CVX tools.

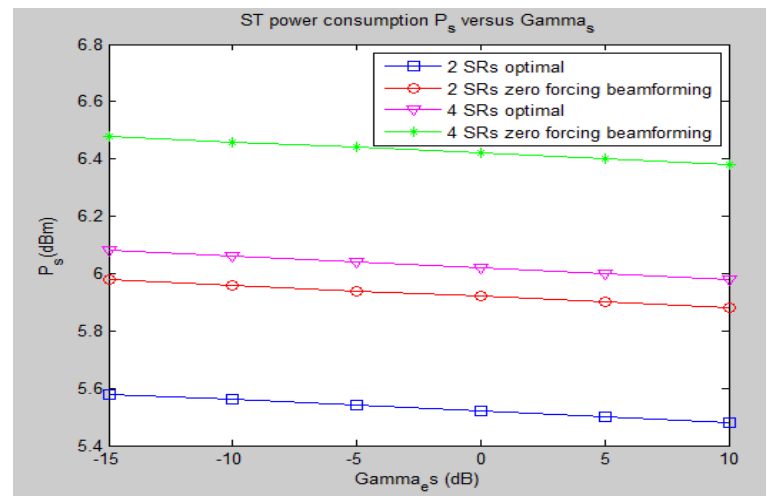

Figure.5 ST Power consumption Ps versus $\gamma_{e, s}$ with $P_{p}=30 \mathrm{dBm}, \gamma_{p}=10 \mathrm{~dB}, \gamma_{e, p}=0 \mathrm{~dB}, \gamma_{s}=30 \mathrm{~dB}$.

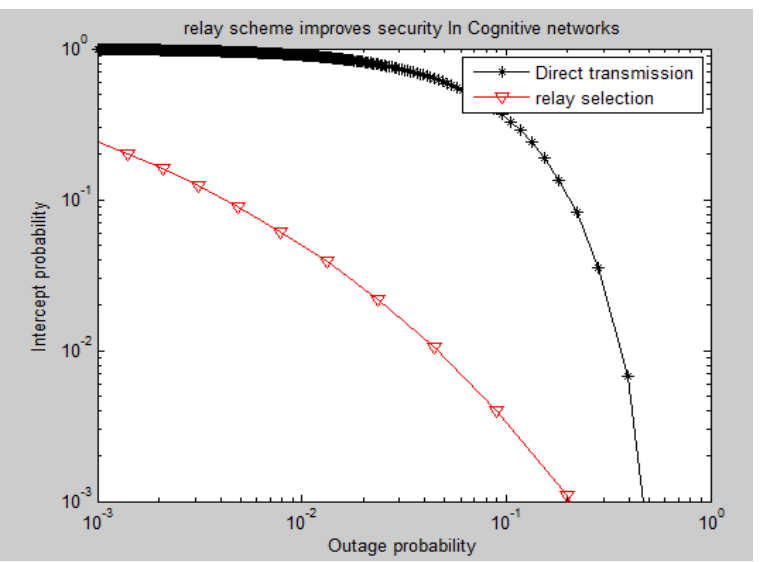

Fig.6 Result of using relay scheme

\section{CONCLUSION}

In this new transmission strategy for SUs to access the spectrum licensed to PUs in CRNs, considering physical layer security for both. The design of beam forming and AN beam forming at ST, aiming to provide PR, Eve, and multiple SRs with different SINR. The problem was formulated as minimizing the power consumption of ST, under different SINR constraints. It can be proved that SDR can be used to convert the initial non- 
convex optimization into a convex SDP and derived. The performanee of the proposed optimat approach is evaluated and compared with computer simulations. The result of simulations had shown that the required value of SINR decreases, power requirement also decreases. In direct transmission, when distance increases, the signal fading also increases. The signal fading is avoided using relay between the users, which results in the reduction of outage probability. The proposed method is simulated and validated with the existing methods to get the optimum results. The performance estimation of the proposed optimal approach is validated and compared using the computer simulations software with an accuracy of $99.8 \%$ of the existing methods.

\section{REFERENCES}

[1] N. Akhtar, F. Arif, and A. M. Siddique,2018. Spectrum Decision Framework to Support Cognitive Radio Based IoT in 5G. Cogn. Radio 4G-5G Wirel. Commun. Syst. 73, 73-92.

[2] Fengchao Zhu and Minli Yao, 2015Improving Physical Layer Security for CRNs using SINR-Based Cooperative Beam forming, IEEE Trans. Vehicular Technology, 56, 13-18.

[3] J. Mitola and G. Q. Maguire, 1999.Cognitive radio: making Software radios more personal, IEEE Personal Commun. Mag., 6, 4, 13-8.

[4] S.Haykin, 2005. Cognitive radio: brainempowered with multiwireless communications,IEEE J. Sel. Areas Commun., 23(2), 201- 220.

[5] Vamvakas P., Tsiropoulou E.E., Papavassiliou S. 2019. On Controlling Spectrum Fragility via Resource Pricing in 5G Wireless Networks. IEEE Netw. Lett. 1:111-115.

[6] N. Zhang, N. Lu, N. Cheng, J. W. Mark, and X. S. Shen, 2013. Cooperative spectrum access towards secure information transfer for CRNs, IEEE J. Sel. Areas Commun., 31(11) 2453-2464.

[7] M. Gastpar, 2007.On capacity under receive and spatial spectrum-sharing constraints, IEEE Trans. Inf. Theory, 63(2) 471-487.

[8] Zhu W., Ma J, Faust 0.2013. A comparative study of different entropies for the spectrum sensing with the techniques. Wirel. Pers. Commun. 69, 1719-1733.

[9] K.B. Letaief and W. Zhan,2009. Cooperative communications for cognitive radio networks, Proc. IEEE, 97(5)878-893.

[10] Xu H., Gao H., Zhou C., Duan R., Zhou X. 2019.Resource Allocation in Cognitive Radio
Wireless Sensor Networks with Energy

[11] O. Simeone, I. Stanojev, S. Savazzi, Y. BarNess, U. Spagnolini, and R. L. Pickholtz,2008.Spectrum leasing to cooperating secondary ad hoc networks, IEEE J. Sel. Areas Commun., 26(1) 203-213.

[12] Stanojev, O. Simeone, U. Spagnolini, Y. BarNess, and R. L. Pickholtz,2010. Cooperative ARQ via auction-based spectrum leasing, IEEE Trans. Commun, 58(6) 1843-1856.

[13] K. Lee, O. Simeone, C. B. Chae, and J. Kang, 2011. Spectrum leasing via cooperation for enhanced physical-layer secrecy, in IEEE ICC. Kyoto, Japan, 2002-2011.

[14] S. Goel and R. Negi,2008 guaranteeing secrecy using artificial noise, IEEE Trans. Wireless Commun., 7(6) 2180-2189.

[15] P.Suresh Kumar, S.Meenakshi, A.Venkatesh.2020.Performance Analysis of High Voltage Intelligent Supervisory Systems Using Neural Networks, Int. J. Adv. Sci. Eng. 6(4) 1525-1532.

[16] Bastami A.H., Kazemi P.2019. Cognitive Multi-Hop Multi-Branch Relaying: Spectrum Leasing and Optimal Power Allocation. IEEE Trans. Wirel. Commun. 18:4075-4088.

[17] Chatterjee S., Maity S.P., Acharya T.2019. Energy-Spectrum Efficiency Trade-Off in the new Energy Harvesting Cooperative Cognitive Radio Networks. IEEE Trans. Cogn. Commun. Netw. 5, 295-303.

[18] P. Suresh Kumar, S. Meenakshi, and G. Prathap.2019.Some Investigations on Heterogeneous Deep LearningNetwork using Stable Election Protocol with Region BasedEnergy Conservation, Int. J. Adv. Sci. Eng. 6(2) 1361-1369.

[19] Khasawneh M., Agarwal A.2014. A survey on security in Cognitive Radio networks; Proceedings of the 2014 6th International Conference on Computer Science and Information Technology (CSIT); Amman, Jordan. 26. 64-70.

[20] S.Meenakshi, P.Suresh Kumar, S.Ramsanjay.2020. Soft Computing Techniques based Digital Adoptive Controllers with Intelligent System for Switched Reluctance Motor, Int. J. Adv. Sci. Eng. 7(1) 1614-1624.

[21] Lai, L.; El Gamal, H.2008. The relayeavesdropper channel: Cooperation for secrecy. IEEE Trans. Inf. Theory, 54, 40054019

[22] M. Dabaghchian, A. Alipour-Fanid, K. Zeng, Q. Wang, and P. Auer2018. Online Learning with Randomized Feedback Graphs for Optimal PUE Attacks in Cognitive Radio Networks. IEEEACM Trans. 
Netw. TON 26. arXiv:1709.

[23] P.Suresh Kumar, S.Meenakshi, 2020. Performance Analysis of FPGA based Implementation of FFT Architecture with Pruning Algorithm for Industrial Applications, Int. J. Adv. Sci. Eng. 7(2), 17701775

[24] Marabissi D., Bartoli G., Fantacci R., Micciullo L.2017. Energy efficient cooperative multicast beamforming in ultradense networks. IET Commun, 12:573-578.

[25] P.Suresh Kumar, S.Meenakshi, G.Prathap, G.Nirmala,2020.Soft Computing Techniques Based MRAC with Intelligent Control for Stability Analysis, Int. J. Adv. Sci. Eng. 6(3) 1430-1438 